\title{
Posibilidades de Combinación del Modelo Cognitivo-Conductual y el Psicoanalítico en el Tratamiento de la Ansiedad
}

\section{Possibilities to Combine Both the Cognitive- Behavioural Model and the Psychoanalitical Model in the Treatment of Anxiety}

\author{
José Manuel Pinto Campos
}

Psicoanálisis Relacional, España

\begin{abstract}
Resumen. El presente artículo propone una complementariedad entre el modelo cognitivoconductual y el psicoanalítico para el tratamiento de la ansiedad que resulta más efectiva que la consideración de cada uno de estos métodos por separado. Se analizan las potencialidades y las limitaciones de ambos tratamientos. Se ofrecen ejemplos clínicos de aplicación del método combinado.

Palabras clave: ansiedad, delirio, verdad subjetiva, distorsión objetiva, integración teórica, pluralismo teórico y combinaciones técnicas.
\end{abstract}

\begin{abstract}
This article aims to propose a unified model for the treatment of anxiety integrating both the cognitive-behavioural and psychoanalytical theories. This approach should be more effective than considering each theory separately. Potentialities and limitations are however evaluated. The present review offers clinical examples where this unified model has been successfully applied.

Keywords: anxiety, delirium, subjective truth and bbjective distortion, theoretical integration, theoretical pluralism, technical combinations.
\end{abstract}

¿En qué se diferencian el tratamiento cognitivo-conductual de la ansiedad y el psicoanalítico? Pensemos en situaciones ansiógenas cotidianas. Por ejemplo, una madre se siente angustiada el primer día que permite salir de noche a su hija de catorce años y, como consecuencia, no puede dormir: se imagina que le pueden hacer daño, robarle o violarla.

Un psicólogo cognitivo-conductual le preguntaría a la madre: ¿cuál es la evidencia de que vaya a ocurrir lo que teme? Después la cuestionaría sobre la probabilidad del suceso y la ayudaría a diferenciar lo posible (la violación) de lo probable. De esta forma, resultaría clara la existencia de distorsiones del pensamiento que le hacen temer lo peor. Y finalmente, buscaría junto a la madre pensamientos alternativos menos catastróficos: el día de nochevieja la calle está mucho más concurrida y el peligro es menor, los padres de una amiga irán a recoger a las menores a la salida de la discoteca, etc.

La correspondencia sobre este artículo debe enviarse al autor a c/ Francisco Silvela 69; 2º D. 28028 Madrid. E-mail: jmpinto@arrakis.es 
Un psicoanalista sería mucho menos directivo, escucharía atentamente a la madre para detectar el origen de la angustia. "La amiga de mi hija es una mala influencia" -podría argumentar la madre-. Ahora el pensamiento asociativo se dirige a enumerar anécdotas en las que se muestra a la amiga como "maligna". Como los prejuicios no se sustentan en hechos relevantes, el psicoanalista señalaría que la madre se siente celosa porque alguien distinto a ella ahora tiene competencia para influir sobre su hija. Además, le diría que está haciendo el duelo por la pérdida de la relación madre-niña, mientras que se van quedando atrás los tiempos en que estaban completamente unidas.

Ambos psicólogos clínicos ayudarían a reducir la ansiedad de la madre, aunque con procedimientos radicalmente distintos. Si las dos orientaciones resultan eficaces, ¿no sería lógico integrarlas? Para ello, se necesita superar el "proteccionismo nacionalista" -el consumo exclusivo de teorías y técnicas de nuestra familia profesional de pertenencia- e importar del extranjero los productos que no se fabrican en nuestro territorio.

\section{El modelo cognitivo-conductual}

La orientación cognitivo-conductual diferencia la ansiedad normal como respuesta adaptativa a una situación de peligro real de la ansiedad patológica debida a miedos imaginarios (Botella y Ballester, 1997; Moreno, 2008). Y el tratamiento se dirige a eliminar los tres componentes de la ansiedad: las reacciones fisiológicas, los pensamientos automáticos y la conducta de evitación.

Para controlar las respuestas fisiológicas se utiliza el aprendizaje de técnicas de relajación. En segundo lugar, el principal método cognitivo es la discusión de los pensamientos patógenos que aparecen en el paciente ante situaciones ansiógenas. Una vez definidos los pensamientos que producen ansiedad, se trata de contrastarlos con la realidad objetiva para detectar las distorsiones cognitivas, los errores lógicos en la manera de pensar del paciente. A juicio de Moreno (2008) los principales errores del pensamiento son: la inferencia arbitraria, el pensamiento catastrófico, el lector de mentes, la personalización, la abstracción selectiva, la sobregeneralización, la magnificación, la minimización, el pensamiento dicotómico y el razonamiento emocional (Moreno, 2008). El último paso es la formulación de pensamientos alternativos más saludables capaces de explicar de una forma más realista la experiencia de la situación ansiógena.

Y en tercer lugar, el método conductual se basa en la exposición -gradual o por inundación, en vivo o en la imaginación- a las situaciones ansiógenas. Así por ejemplo, para curar un caso de agorafobia se puede pedir al paciente que detalle una lista de situaciones temidas ordenadas según el grado de ansiedad producido y se pide al paciente que las vaya afrontando paulatinamente empezando por la que menor ansiedad le provoque. La superación de la evitación produce la eliminación de la ansiedad por habituación. A corto plazo, la evitación se refuerza a sí misma porque elimina la ansiedad. Pero a medio plazo, produce una disminución de la confianza para aprender a controlarla.

El modelo cognitivo conductual es una mezcla de sentido común refinado (relajación, métodos distractivos, enfrentamiento graduado a las situaciones ansiógenas) y de aplicación del método científico: se realiza un contraste de hipótesis para poner de manifiesto la irracionalidad y la falsedad de los pensamientos que producen la ansiedad. Veámoslo en el tratamiento del trastorno de pánico que proponen Botella y Ballester (1997).

\section{DISCUSIÓN COGNITIVA DE LOS PENSAMIENTOS CATASTRÓFICOS}

1. Definición del pensamiento negativo. ¿Qué temo? ¿Qué creo que me puede suceder? A veces, no está claro cuál es el pensamiento anterior a la respuesta de ansiedad. Concretar estos pensamientos producen una sensación de control y permite poner a prueba estas creencias.

2. Análisis de las evidencias a favor y en contra. ¿Qué pruebas objetivas tengo de que lo que temo vaya a ocurrir? 
3. Análisis de probabilidades. ¿Qué probabilidad hay de que se cumpla el pensamiento catastrófico?

4. Interpretaciones alternativas. ¿Existen otras explicaciones alternativas para entender lo que me está sucediendo?

5. Desdramatización. ¿Qué sería lo peor que podría sucederme si lo que temo fuera cierto? ¿Sería tan horrible?

6. Utilidad del pensamiento. ¿Me ayudan estos pensamientos o me ponen más ansioso?

Apliquemos este esquema de trabajo a un caso clínico. Vicente era un paciente de cuarenta años que vino a consulta a consecuencia de padecer un intenso temor a ir a la cárcel. Se enfrentaba a un juicio penal en el que el fiscal le acusaba de tenencia y difusión de archivos informáticos de pederastia y la policía aportaba las pruebas de la posesión de estos archivos en su ordenador.

La realidad de lo ocurrido era completamente distinta. Vicente era un adicto a la pornografía. Cuando se colaba alguna vez un archivo de abuso de menores lo destruía inmediatamente porque el paciente no era en modo alguno un paidófilo. Pero como realizaba descargas masivas de estas películas y las almacenaba sin revisarlas previamente, se encontró de súbito con la acusación de la policía que había detectado archivos pederastas en su ordenador. A continuación se transcribe un diálogo con el paciente:

P- Si me condenan a más de dos años mi vida actual se arruinaría, perdería el trabajo, no podría pagar la letra del piso y me hundiría en la cárcel.

T- ¿Qué razones tienes para pensar que puedas ir a la cárcel?

$\mathrm{P}$ - La justicia es muy arbitraria. Continuamente aparecen en las noticias casos en los que un corrupto se salva de ser condenado a prisión.

T- Esos son casos contrarios al tuyo. Se trata de culpables que eluden la justicia. Tu eres un inocente que temes que te sentencien como culpable. ¿Qué noticias conoces de inocentes que hayan ido a la cárcel?

P- No me acuerdo de ningún caso.

T- Por tanto, fíjate que no hay evidencias de tu miedo. Por tanto, se trata de una inferencia arbitraria sin razones reales. ¿Qué más temes?

P- Temo que puedan demostrar que difundí estos archivos a otros ordenadores.

T- Esa es la acusación del fiscal. Sin embargo, la policía no ha demostrado este punto y no te acusa de difusión. Por otra parte, el informe del perito informático que va a presentar el abogado defensor demuestra que el programa de descargas permite la transferencia automática de estos archivos a otros ordenadores. Como verás estas teniendo un pensamiento erróneo en el que maximizas la acusación del fiscal y minimizas las pruebas de la defensa. ¿Qué probabilidad crees que tiene tu temor de ser cierto?

P- Un uno por cierto. Pero esto basta para que esté nervioso. Realmente es un temor residual. La mayoría de mi mente piensa que no voy a ir a la cárcel. De lo que sí estoy seguro es de que no tengo defensa en cuanto a la tenencia de archivos. Esos archivos estaban en mi ordenador, eso es un hecho objetivo que no puedo negar. Si el fiscal quiere negociar y me condena a seis meses de cárcel creo que aceptaría el trato con tal de no arriesgarme a que en el juicio me condenen a una pena mayor.

T- No estás teniendo en cuenta que eres inocente, tú eras inconsciente de la existencia de esos archivos. Además, cuentas con mi testimonio en donde demostraré que tu estructura de personalidad no es perversa.

P- Lo pensaré.

En resumen, la potencia del modelo cognitivo-conductual para el tratamiento de la ansiedad reside en la sustitución de la oscuridad de la emoción irracional del paciente por la iluminación de la razón más próxima a la realidad objetiva. 
Y las limitaciones de este modelo son también las limitaciones de la razón objetiva. En muchas ocasiones el mundo emocional que produce la respuesta ansiosa no es comprensible con la lógica de la razón científica. En estos casos, sólo se puede luchar contra las raíces profundas de la ansiedad si se comprende la lógica emocional basada en los modelos relacionales que han construido la personalidad del paciente y que estudia el psicoanálisis.

Así, por ejemplo, Vicente hablaba en la escena antes expuesta de una ansiedad residual que le torturaba con frecuencia y que no podía controlar pese a saber que era muy poco probable. Para reducir esta angustia no vale la fuerza de la razón científica -el modelo cognitivo-conductual- sino que es necesario hacer uso de la orientación psicoanalítica. En este sentido, se necesitó superar el hábito que tenía de "ponerse en lo peor", un patrón de conducta basado en la relación que tenía con su madre, una mujer perfeccionista que le señalaba todos sus errores.

\section{El modelo psicoanalítico}

A diferencia del modelo cognitivo-conductual, la tradición psicoanalítica sostiene que no todas las ansiedades son conscientes, sino que algunas son inconscientes. En el primer caso se trata de trabajar sobre la ansiedad manifestada por el paciente y en el otro, de descubrir la ansiedad latente que está escondida tras el síntoma. Veamos cómo funciona esta segunda concepción en el famoso caso Juanito de Freud sobre una fobia a los caballos:

"El miedo a la castración se dirige a un objeto distinto y toma una expresión disfrazada -ser mordido por un caballo en lugar de ser castrado por el padre-. La formación sustitutiva tiene dos evidentes ventajas. En primer lugar evita un conflicto por ambivalencia, pues el padre es al mismo tiempo un objeto amado; y en segundo, permite al yo terminar el desarrollo de la angustia. La angustia de la fobia es, en efecto, condicional. No aparece sino ante la percepción de su objeto, cosa perfectamente justificada, puesto que sólo entonces existe el peligro. De un padre que no está ahí no puede temerse la castración. Ahora bien, el padre no puede ser suprimido, aparece ante el sujeto cuando quiere. Pero una vez sustituido el padre por un animal, el sujeto no tiene mas que evitar la percepción de este último, o sea, su presencia, para vivir libre de peligro y de angustia. Así pues, Juanito impone a su yo una limitación: la de no salir a la calle para no encontrarse con un caballo... Tal sustitución tendría la ventaja de que el sujeto podía asegurarse contra el peligro exterior apelando a la fuga y evitando la percepción, mientras que contra el peligro interior no hay fuga posible".

(Freud, 1926)

El psicoanálisis contemporáneo ya no considera a la sexualidad como la única motivación profunda de la que derivan todas las demás. Hoy en día se trabaja sobre un conjunto de motivaciones básicas: la autoconservación, el apego, el narcisismo, la sexualidad y la agresividad (Bleichmar, 1997). Sin embargo, si se elimina la explicación omnicomprensiva -el miedo a la castración o el miedo a deseos libidinales que están reprimidos- para la comprensión de las fobias y otras neurosis, el esquema del pensamiento de Freud sigue estando vigente. En ocasiones, la angustia sintomática serviría para amortiguar y encubrir ansiedades de base mucho más intensas que resultan inconscientes.

Posteriormente, en la tradición kleiniana (Segal, 1984), la ansiedad ha tenido un lugar central tanto en la teoría como en la clínica. Las posiciones esquizo-paranoide y depresiva se teorizan a partir de la ansiedad predominante, y la técnica reside en la interpretación del conjunto formado por la ansiedad y los mecanismos de defensa.

Sin embargo, los kleinianos no pretenden eliminar la ansiedad como ocurre en la tradición cognitivo-conductual. En mi opinión, para ellos la ansiedad del paciente es el motor del tratamiento, una energía que no se pierde, sólo se transforma en otra forma de ansiedad más evolucionada y saludable. 
Así, en la posición esquizo-paranoide, la ansiedad predominante es que el objeto persecutorio aniquile al objeto ideal. Para reducir esta ansiedad se utilizan mecanismos de defensa: escisión, proyección, introyección e identificación proyectiva. Pero, a su vez, estos mecanismos de defensa producen nuevas ansiedades. La proyección crea ansiedad persecutoria; la introyección, ansiedad hipocondríaca; y la identificación proyectiva, ansiedad de retaliación y de ser controlado por el objeto. Y si todo marcha bien -si las experiencias buenas superan a las malas- la ansiedad paranoide se transforma en ansiedad depresiva.

Como en el caso de los kleinianos, Winnicott $(1961,1965,1969,1971)$ trata las ansiedades esquizoides y depresivas, así como las angustias normales y conscientes (ansiedad de separación, miedo al abandono, etc.). Pero Winnicott presenta la particularidad de enfrentarse a la angustia más profunda e impensable, el miedo al derrumbe psíquico, el terror a la locura, y lo hace con una genial originalidad: no pretende eliminar ni esquivar la ansiedad, sino que el paciente la reexperimente ya que no puede recuperarla mediante el recuerdo:

"A veces es un alivio que acontezca la tragedia y el paciente enloquezca, pues si se da cabida a una recuperación natural, habrá "recordado" hasta cierto punto la locura original... Se apreciará que si en un caso así se pretende abordar el derrumbe con una urgencia psiquiátrica de curar, la finalidad de aquél se pierde por completo, ya que al derrumbarse el paciente perseguía un fin positivo y su colapso no es tanto una enfermedad como un primer paso hacia la salud... La cura sólo sobreviene si el paciente puede llegar hasta la angustia en torno de la cual se organizaron las defensas... Sólo curará si alcanza el estado de derrumbe original”.

(Winnicott, 1965)

Quizás no pueda encontrarse una mayor valentía en un psicoanalista: alguien que se enfrenta serenamente a las escenas más temidas de un tratamiento de psicoterapia. Aparte del suicidio, el máximo miedo de un psicólogo clínico es que el paciente enloquezca, se derrumbe psíquicamente y necesite ser internado en un centro psiquiátrico.

Winnicott no teme el desmoronamiento del paciente, el derrumbe psíquico que lo suma en un estado de absoluta dependencia respecto del terapeuta; confía en su capacidad de sostenimiento (holding) para poner en marcha los procesos madurativos del desarrollo que conducirán a la cura del paciente. Sin duda, Winnicott es un psicoanalista muy atractivo que enamora a muchos de sus lectores.

Pero pasado un tiempo hay que convertir el embelesamiento en amor real: mezclar la fascinación del principio con los defectos que se le van detectando. Y uno de los defectos de Winnicott le viene de familia. El psicoanálisis enseña al analista a contener la angustia del paciente, pero carece por completo de medios prácticos para favorecer el que aprenda a controlar la ansiedad por sí mismo. Como las bases del psicoanálisis promueven una técnica no directiva, según la cual el terapeuta analiza al paciente evitando caer en el rol de profesor, enseñar el autocontrol de la ansiedad debe ser un producto que hay que importar de la orientación cognitivoconductual cuya tradición sí acepta explícitamente enseñar al paciente modelos de conducta más saludables y adaptativos.

Por una parte, hay una larga tradición en psicoanálisis para formar a los terapeutas en la habilidad de reducir la ansiedad del paciente. Para ello, se han utilizado varios conceptos técnicos: la contención de Bion (1991), el sostenimiento (holding) de Winnicott (1964) y la terceridad de Benjamin (2004). Para esta última autora la contención de la angustia del paciente requiere que el terapeuta pueda adoptar una tercera posición en la que se identifique simultáneamente con la parte infantil del paciente y con su propia parte adulta. El psicoanalista debe funcionar como una buena madre empática con su hijo -por ejemplo, cuando se compadece del llanto del niño que se ha hecho una herida por caerse al suelo- y, al mismo tiempo, poder diferenciarse de él y ofrecer una versión de los hechos menos catastrófica -asegurándole que el dolor se le pasará-.

Y por otra parte, esta misma tradición perpetúa los roles asimétricos de la psicoterapia: un paciente-niño-enfermo y un terapeuta-adulto-sano. Se crea una relación de dependencia del paciente hacia el terapeuta que se alarga a través de toda la terapia. Por el contrario, la tradición cognitivo conductual promueve la independencia del 
paciente a través de enseñarle el autocontrol de la ansiedad. La solución ideal sería combinar ambos métodos: alentar la independencia y buscar la dependencia en relaciones de intimidad en donde el paciente se atreva a expresar sus angustias y dejarse apaciguar. Para ilustrar estas ideas expongo a continuación una viñeta clínica.

Luis era un paciente de cuarenta y cinco años que había descubierto un método propio de combatir la ansiedad. Su estrategia era realizar un diálogo interno y continuo entre la parte frágil (F) de la personalidad que sufría con sentimientos y pensamientos ansiógenos y la parte más madura (M) que lo auto-apaciguaba. Así, cuando le cambiaron de puesto de trabajo en su empresa de catering para suministrar las comidas a colegios, tuvo el siguiente diálogo interno:

M- ¿Qué temes en realidad?

F- Equivocarme en algún pedido y que le falten menús a algún colegio.

M- Entonces, puedes hacer el trabajo y luego pedir a algún cocinero con más experiencia que te supervise por si tienes algún error. ¿Qué más miedos tienes?

F- Que se enfade el jefe: se comporta conmigo como un enemigo, me ha dejado solo para un trabajo para el que no estoy cualificado.

M- En realidad, el jefe no es tu enemigo: ha confiado en ti para un trabajo más difícil. Lo que ocurre es que quieren hacer el trabajo con menos personal. Además, ayer le pediste ayuda y trabajó contigo un par de horas. ¿Qué más miedos tienes?

F- Que me despidan por hacerlo mal.

M- Es lógico que tengas ese miedo porque la empresa ha perdido clientela. Pero de momento hay trabajo para ti. Además, si llegara la desgracia de perder tu empleo, sabes que tienes el piso pagado, que eres muy austero y que tu mujer trabaja.

F- Yo no quiero trabajar en esto, no estoy preparado. Voy a hablar con la dirección para que me vuelvan a cambiar a mi puesto anterior.

M- No hace falta que te alarmes. Puedes dividir la actividad de un día en partes. Céntrate sólo en el pedido que tienes entre manos, en el paso que tienes que dar. Así te parecerá más fácil porque tu ansiedad será más pequeña.

El método de Luis es una mezcla de reestructuración cognitiva (análisis de las distorsiones del pensamiento y búsqueda de pensamientos alternativos no catastróficos) y del logro de la terceridad psicoanalítica (puede identificarse, al mismo tiempo, con la parte ansiógena-infantil y con la parte auto-apaciguadora-adulta). La originalidad de Luís estribaba en que todo esto lo había descubierto por sí mismo. ¿Qué parte del autocontrol de la ansiedad se debía a la creatividad de Luís? ¿Y qué parte se debía a la acción implícita de la psicoterapia?

Luis había nacido ciego de un ojo debido a un glaucoma y sólo tenía una visión reducida en el otro. Ya en su primera infancia tuvo que ser sometido a diversas operaciones para no perder más visión en el ojo sano. Estos hechos motivaron que los padres estuvieran permanentemente angustiados por el temor a que la minusvalía de su hijo se agravara. Así, le educaron de forma sobreprotectora y le obligaban a volver a casa nada más terminar el colegio, de manera que le impedían relacionarse con sus compañeros. Además, le desanimaban a realizar cualquier actividad nueva que representara un peligro real o imaginario.

Estas experiencias infantiles constituían tanto la base de su ansiedad de adulto como la causa de su personalidad extremadamente dependiente. En cada reto que tenía que afrontar, y que le despertaba angustia, esperaba que su cuidador se lo solucionara. Actitud que resultó determinante para que su mujer se divorciara de él: se quejaba, con razón, de que Luís fuera muy pasivo y quisiera vivir exclusivamente en el entorno seguro de sus rutinas domésticas.

Tras el divorcio, Luis comenzó una psicoterapia conmigo porque se sentía culpable del fracaso de su matrimonio y quería cambiar. Con el transcurso del tiempo se fue haciendo consciente de su evitación de las situaciones de ansiedad y de cómo este rasgo de su carácter le creaba una minusvalía psíquica para luchar contra las 
dificultades cotidianas. Luís decidió evolucionar poco a poco, planteándose la meta de "ser capaz de" realizar las actividades que le daban miedo: preparar un viaje, enfrentarse a un trabajo nuevo, ligar con una mujer o simplemente cocinar y limpiar la casa.

La contribución original de Luis al método integrado de control de la ansiedad era la habilidad de convivencia pacífica entre dos mitades de su personalidad: la parte antigua que sufría de ansiedad generalizada y la parte nueva que se enfrentaba a sus miedos. Luís no olvidaba nunca su parte infantil miedosa, no la combatía, le producía ternura. Pero tampoco se dejaba dominar por ella.

Hay que tener en cuenta que esta convivencia pacífica no es la norma en psicoterapia. Lo más frecuente es el conflicto entre diferentes partes de la personalidad, la guerra por la dominación de un sector sobre el otro, y la incapacidad de llegar a soluciones consensuadas.

Por otro lado, la contribución de la psicoterapia a la creación del método anti-estrés se cimienta en la persecución de dos objetivos distintos: trabajar sobre el pasado para descubrir sus angustias más profundas -el miedo a la minusvalía, quedarse totalmente ciego- y luchar en el presente por el desarrollo de sus capacidades a pesar de la fuerte resistencia a abandonar el patrón de conducta dependiente. De esta forma, se potenciaba tanto la parte infantil como la parte adulta del self. Un tratamiento pretendidamente más profundo que hubiera referido todos los conflictos presentes al pasado hubiera sido iatrogénico, puesto que Luís ya se sentía discapacitado, y lo nuevo era precisamente superar la minusvalía psíquica.

Esta apertura a lo nuevo, al fortalecimiento de la parte adulta -la parte cuidadora de uno mismo, la parte auto-tranquilizante- de la personalidad es indispensable para el aprendizaje del auto-control de la ansiedad. Desgraciadamente, la mayoría de las teorías psicoanalíticas -aunque en la práctica clínica no ocurre siempre así- han olvidado este objetivo. Se necesita ampliar la metáfora de la "profundidad" como el mayor logro en psicoterapia con una nueva metáfora tridimensional. Lo óptimo no es sólo lo más profundo (la infancia, las raíces) sino también lo más ancho y alto (la madurez, el tronco y las ramas).

Mitchell (1988) ha criticado a los psicoanalistas de la "detención del desarrollo" (Balint, Winnicott, Kohut, etc.) por infantilizar a los pacientes. Para ellos la patología severa es el resultado de un suministro inadecuado de necesidades primarias, que se curan mediante la provisión de funciones maternas que producen en el paciente-niño experiencias no conflictivas que le permiten reemprender el crecimiento de su personalidad. De ahí se deriva una técnica de intervención en donde se confunden las necesidades infantiles con las necesidades adultas:

"Referir las diferentes transformaciones de las necesidades del paciente a sus primeras manifestaciones infantiles, equivale a distorsionar seriamente su significado e infantilizar al mismo tiempo al analizando".

(Mitchell, 1988)

Podemos ilustrar esta idea con un ejemplo clínico. Un paciente joven con un gran talento literario quiere escribir un libro de poesías pero está bloqueado en el intento porque se deja llevar por sus necesidades infantiles de grandiosidad. Se imagina el libro futuro como el medio de llegar a ser famoso y rico, y estas fantasías le aumentan el nivel de exigencia de manera que redacta con la rapidez de una tortuga. Las necesidades infantiles de grandiosidad no tienen nada que ver con sus necesidades adultas: es perfectamente capaz de escribir ese libro, le frena el perfeccionismo y la falta de auto-disciplina. En este caso, alimentar el self grandioso como propone Kohut es iatrogénico. Mi práctica clínica es justo la contraria. Mostrar al paciente cómo esas necesidades infantiles de convertirse en un rico famoso le obstaculizan a la hora de conseguir la satisfacción de su necesidad adulta de publicar su libro.

\section{Las bases para un modelo combinado}

El modelo cognitivo-conductual diferencia nítidamente entre la ansiedad beneficiosa y adaptativa, que se 
dirige al control de una situación peligrosa objetivamente, y la ansiedad perjudicial y patológica producida por temores imaginarios.

Sin embargo, esta distinción dicotómica entre verdad objetiva y distorsión patológica impide el tratamiento de muchos trastornos (delirios psicóticos, neurosis traumática, anorexia, etc.) donde el paciente no puede aceptar sus errores de pensamiento y sus distorsiones objetivas porque cree firmemente en que su ansiedad es completamente real. Si el terapeuta insiste en contradecir al paciente y proclamar que él es el poseedor de la verdad, el paciente no se siente entendido y se resquebraja la alianza terapéutica. Veamos cómo explica esta crítica un paciente que había sido tratado por un psicólogo cognitivo-conductual antes de acudir a mi consulta:

"Los cognitivo-conductuales tienen un error de base. La reestructuración cognitiva que proponen es un planteamiento exclusivamente racional. Yo estoy disociado entre lo que pienso y lo que siento. Cuando pienso que me voy a morir me traslado a un escenario donde soy completamente vulnerable como en mi niñez y entonces sólo cuenta la emoción, no las ideas racionales. El tratamiento cognitivo-conductual se relaciona exclusivamente con la parte adulta de la personalidad. A mí me crea paranoia. Me hace sentir que no pienso bien. Te dicen: "tú no sabes pensar". Esto me genera angustia y alimenta al niño angustiado que llevo dentro".

¿Cómo resolver el dilema entre la verdad objetiva representada por el terapeuta y las creencias contrarias del paciente? ¿Tiene el terapeuta que aceptar la posición del paciente de creer en su delirio y darle la razón o renunciar a la evidencia de que la anoréxica que se ve gorda está en realidad esquelética? No, el terapeuta debe convivir democráticamente durante un buen tramo de la psicoterapia con dos posiciones divergentes. Podrá decir al paciente que "no cree en sus ansiedades persecutorias" aunque para el paciente sean absolutamente verdaderas o bien que "piensa que está muy delgada" a pesar de que ella se vea gorda. Esta aceptación de los dos puntos de vista contrarios es suficiente para que el paciente colabore en la terapia. Un error grave sería imponer al paciente por la fuerza nuestra verdad.

Para resolver este impasse se necesita introducir un concepto intermedio entre objetividad y distorsión: la verdad subjetiva del paciente derivada de las experiencias reales de su pasado. La verdad subjetiva de la ansiedad patológica es una verdad objetiva porque ocurrió realmente en el pasado. Y es también una falsedad objetiva, ya que no está vigente en la actualidad. Para ilustrar la tensión entre verdad subjetiva y distorsión objetiva se presenta a continuación una nueva escena clínica.

Raúl era un hombre de 55 años que vino a consulta a causa de una depresión mayor tras su divorcio. Su mujer se había separado de él sin que hubiera mediado conflicto conyugal alguno: simplemente deseaba ser más libre. El valor principal de Raúl era el amor a su mujer y a sus dos hijos, de manera que el divorcio lo hundió en una depresión profunda acompañada de una angustia que no cesaba desde la mañana hasta la noche. En las sesiones se centraba una y otra vez en su perplejidad por encontrarse solo después de treinta años de haber vivido feliz en su matrimonio.

Después de un año de tratamiento recibió la noticia de que su exmujer se había vuelto a emparejar. Esto terminó de hundirlo definitivamente. Sentía que se había roto por completo su familia y declaraba que se suicidaría cuando su hijo de trece años creciera y ya no lo necesitara más. Yo le decía que estaba confundiendo la pérdida de su exmujer con la pérdida de sus dos hijos que nunca se separarían de él. Estas razones no le servían y la ansiedad se disparó hasta el punto de no poder dormir por las noches ni siquiera con medicación.

Afortunadamente, la situación cambió notablemente después del verano. Pasó un mes con sus hijos y se sintió feliz con ellos. Esto provocó que la angustia se eliminara casi totalmente, aunque persistía la sintomatología depresiva. Es decir, necesitó la prueba de realidad consistente en comprobar que no perdía a sus hijos para poder vencer así la ansiedad. La verdad subjetiva era una verdad objetiva ya que su mujer había desaparecido de su vida, pero era también una distorsión objetiva puesto que confundía la pérdida de su exmujer con la pérdida de "toda" su familia. 
En resumen, la polémica sobre qué tratamiento es mejor para combatir la ansiedad -el psicoanalítico o el cognitivo conductual- no tiene en cuenta que ambos métodos son efectivos. El modelo psicoanalítico es mejor para descubrir la verdad subjetiva que subyace a la ansiedad y el modelo cognitivo-conductual es superior para detectar y combatir las distorsiones del pensamiento.

Otra fuente de polémica es definir qué método de tratamiento es mejor para un trastorno determinado. Así, mis colegas conductistas parten de la superioridad del modelo cognitivo-conductual en el tratamiento de las fobias, las crisis de pánico, la agorafobia y la ansiedad generalizada, lo que les lleva a concluir que este modelo es siempre mejor que el psicoanalítico en todos los trastornos de ansiedad. Sin embargo, en mi opinión, cuando se toman otros prototipos de la ansiedad más complejos el modelo cognitivo-conductual deja de ser efectivo en la primera fase del tratamiento.

En mi experiencia clínica, este es el caso de los delirios psicóticos. Aquí se da la máxima distancia entre la verdad subjetiva del paciente y la distorsión objetiva que puede apreciar cualquier persona normal. En estas circunstancias, señalar los errores de pensamiento no sirven de nada durante el primer tramo de la psicoterapia que puede durar años: el paciente no opone ninguna resistencia a sus creencias delirantes. Veámoslo más concretamente en el caso clínico que expongo a continuación.

Antonio, un hombre de cuarenta años, vino a consulta a consecuencia de padecer de ansiedad persecutoria. Uno de sus delirios más frecuentes era que una multinacional le impedía trabajar porque disponía de fuentes de datos secretas que reflejaban las culpas de su pasado, por ejemplo, el hecho de que se hubiera acostado con prostitutas en su juventud. Otro delirio se centraba en imaginar que una organización muy poderosa se introducía en su ordenador con la intención de controlarle y matarle.

La paranoia se desencadenó cuando tenía veinticinco años. Se encontraba realizando un curso de formación profesional y empezó a pensar que los profesores le hablaban en clave: extraía una frase de su contexto y buscaba interpretaciones persecutorias en su contra. Una de las ideas que imaginaba era que la empresa de formación tenía documentos que atestiguaban que él había abusado de un niño siendo adolescente. Como no soportaba esta culpabilidad confesó a un profesor que había hecho cosas malas en su pasado. Al final, tuvo que ser internado en un centro psiquiátrico durante unos días debido a una crisis aguda de ansiedad.

La culpa persecutoria se basaba en tres acusaciones principales. La primera era que cuando tenía catorce años, un niño de diez años le hizo una felación. La segunda era haber asistido durante un par de semanas a la secta de la Cienciología. Y la tercera era haberse ido con prostitutas.

A Antonio le tranquilizaba que le dijera que no había habido abuso de menores puesto que él no era un adulto y los dos eran niños. Y también le apaciguaba escuchar que tener relaciones sexuales con prostitutas en alguna ocasión es un hecho muy corriente. A pesar de todo ello, las ideas paranoicas se mantenían intactas.

En realidad, sus perseguidores eran la proyección condensada de figuras de su infancia. Hasta los catorce años asistía todas las semanas a reuniones religiosas en las que terminaba confesándose con un cura tutor muy severo. Una vez que abandonó la religión, una parte disociada de sí mismo se identificó con este cura cruel que conocía su mente a la perfección. Por otra parte, Antonio vivía en un barrio obrero rodeado de amigos marginales que se dedicaban al tráfico de drogas y que, en ocasiones, habían ido a parar a la cárcel. Con el paso de los años se separó de ellos, pero temía encontrárselos por el barrio y que pudieran vengarse de él.

En la primera fase de la psicoterapia no se aceptan pensamientos alternativos que se opongan a la ansiedad delirante. Una crítica frontal no sirve de nada y es contraproducente. Si se argumenta que no hay ninguna organización que espíe su ordenador para extraer información confidencial, el paciente responderá con noticias de periódicos en donde estos hechos sí han tenido lugar.

Por tanto, el primer objetivo era, tan solo, descubrir su verdad subjetiva, legitimar su ansiedad persecutoria en base a las experiencias de su infancia y juventud. Posteriormente, como segundo objetivo, intentaba fortalecer tanto su parte infantil como su parte adulta. En el lado infantil, buceamos en las experiencias paranoicas con una madre muy primaria, continuamente crítica y persecutoria. En el lado adulto, nos enfrentamos a la consolidación de su trabajo autónomo muy precario y a la construcción de nuevas relaciones de amistad. Le mos- 
traba cómo su carácter socavaba algunas de sus relaciones: cuando le venían los pensamientos paranoicos respecto de un amigo o un cliente, se distanciaba defensivamente y rompía el vínculo.

Pasados dos años de psicoterapia psicoanalítica, los delirios fueron reduciendo su frecuencia para instalarse sólo en los momentos en que el paciente se encontraba más débil. Antonio podía ya criticar parte de la paranoia y encontrar con mi ayuda pensamientos alternativos: por fin, se podía utilizar el método cognitivo.

Sin embargo, de tanto en tanto, volvía a sufrir crisis de ansiedad persecutoria en donde se le desencajaba la cara y la ansiedad era intensísima y no encontraba antídotos que lo tranquilizaran. Una de estas crisis ocurrió cuando el paciente se encontraba muy solo en Alemania buscando trabajo. En una ocasión le presentaron a una persona que se dedicaba a cuidar a enfermos mentales en una clínica psiquiátrica. Inmediatamente, Antonio tuvo miedo de que le descubriera y pudiera pensar que estaba loco. Y de ahí volvió a torturarse con la idea de que la multinacional en donde esperaba encontrar trabajo conociera que se había acostado con prostitutas en su juventud y no le contratara.

Para estas fechas, mi intención era que él fuera capaz de administrarse por sí solo el tratamiento cognitivo:

T- Tienes que hacer por ti mismo lo que he hecho contigo otras veces para reducirte la ansiedad. Hazte tres preguntas: ¿qué probabilidad hay de que suceda lo que temo?, ¿qué pensamientos alternativos pueden explicar mejor la realidad?, y si ¿sería tan horrible que sucediera lo que temo? Pensémoslo en relación con la última crisis que tuviste.

P- Creo que la probabilidad de que la multinacional me haya espiado es del $60 \%$.

T- ¿Qué hipótesis alternativas se te ocurren?

P- Yo soy una currito de a pie, no un directivo. Lo lógico sería que espiaran a personas importantes como ocurre con los políticos que salen en la prensa por corrupciones que destapan los periodistas.

T- Además, fíjate que los hechos pasados que temes que conozcan han ocurrido hace más de veinte años. ¿Cómo iban a ser tan previsores de manera que te espiaran cuando sólo eras un joven?

P- Vamos a bajar la probabilidad a un $30 \%$.

T- Y si ocurriera que no te contrataran, ¿sería tan catastrófico?

P- No me pasaría nada en la práctica porque siempre he trabajado en empresas pequeñas. Pero perdería la ilusión de estar dentro de una gran empresa.

Una vez que Antonio fue utilizando por sí mismo este método, le sugerí que ya estaba en condiciones de auto-controlarse la ansiedad por medios cognitivos y, por tanto, no necesitaba la medicación antipsicótica, salvo como recurso de urgencias cuando tuviera una crisis

Ahora podíamos combinar el tratamiento cognitivo-conductual con el psicoanalítico: liberarse de los modelos relacionales persecutorios que había mantenido con una madre hipercrítica, con un cura de la "Inquisición" y con unos amigos delincuentes.

La ansiedad manifiesta ocultaba la ansiedad más profunda por un superyó tiránico que le exigía la pureza de un santo o bien le atacaba cruelmente. ¿Qué ventaja se obtiene con esta ocultación? La ventaja es que se sustituye un perseguidor interno (el superyó) por otro externo (la institución poderosa). Como argumentaba Freud (1926), ante el perseguidor interno no hay escapatoria posible; sin embargo, ante el perseguidor externo cabe la defensa de la huída y la evitación.

\section{Conclusiones}

El psicoanálisis (Ps) y la orientación cognitivo-conductual (C-C) difieren en la teoría y la técnica para el tratamiento de la ansiedad. Para los C-C la ansiedad es un trastorno en sí misma que hay que tratar con urgencia. Sin embargo, desde el lado de los Ps se aprecia, en ocasiones, un olvido a tratar directamente la ansiedad a la 
que se considera como síntoma, no como causa de enfermedad. La angustia se valora como una señal que nos orienta en la comprensión de los conflictos latentes e inconscientes del paciente, de manera que una dosis de ansiedad tolerable resulta ser un apoyo de la alianza terapéutica. Así, el psicoanálisis se ha especializado en tratar las angustias de base y la orientación cognitivo-conductual, en controlar y eliminar las ansiedades sintomáticas.

Las técnicas de ambas orientaciones son radicalmente distintas. Los $\mathrm{C}-\mathrm{C}$ emplean una mezcla de método empírico (conductismo) y racional (cognitivismo), mientras que los Ps utilizan la lógica emocional, tratan de analizar los patrones relacionales patógenos que están en la raíz de la ansiedad.

Además, la relación con el paciente también es muy diferente. Los C-C tienen por objetivo el que el paciente aprenda el auto-control de la ansiedad, de manera que funcionan como profesores con buena pedagogía. Sin embargo, los Ps tienen prohibido ser directivos y, por tanto, se centran sólo en contener la angustia del paciente, no en enseñar esa habilidad.

En conclusión, hoy en día no es posible una integración entre los modelos C-C y Ps ni en el nivel teórico ni técnico. Sin embargo, sí es posible una complementariedad de ambos modelos y, como he pretendido mostrar en este artículo, esta combinación resulta más eficaz en el tratamiento de la ansiedad que el uso de cualquiera de los dos métodos por separado.

\section{Referencias}

Benjamin J. (2004). Más allá de la dualidad agente-paciente: una visión intersubjetiva del tercero. Revista Intersubjetivo, 6, 7-38.

Bion W. R. (1991). Apendiendo de la experiencia. México: Ediciones Paidós.

Bleichmar H. (1997). Avances en psicoterapia psicoanalítica. Madrid: Ediciones Paidós

Botella C. y Ballester R. (1997). Trastorno de pánico: evaluación y tratamiento. Madrid: Ediciones Martínez Roca.

Freud S. (1926). Inhibición, síntoma y angustia. Obras completas. Tomo VIII. Madrid: Biblioteca Nueva.

Mitchell S. (1988). Conceptos relacionales en psicoanálisis. México: Siglo XXI.

Moreno P. (2008). Superar la ansiedad y el miedo. Bilbao: Desclée de Brouwer.

Pinto J. M. (2001). El desarrollo del ideal del yo. Revista Intersubjetivo, 3, 178-198.

Segal H. (1984). Introducción a la obra de Melanie Klein. Barcelona: Ediciones Paidós.

Winnicott D. (1971). Sostén e interpretación. Barcelona: Editorial RBA.

Winnicott D. (1969). Individuación. Barcelona: Editorial RBA.

Winnicott D. (1965). La psicología de la locura. Una contribución psicoanalítica. Barcelona: Editorial RBA. Winnicott D. (1964). El juego del garabato. Barcelona: Editorial RBA.

Winnicott D. (1961). Nuevas observaciones sobre la teoría de la relación parento-filial. Barcelona: Editorial RBA.

Manuscrito recibido: $12 / 02 / 2014$

Revisión recibida: 13/06/2014

Manuscrito aceptado: 16/06/2014 